\title{
Serum Biomarkers in Valvular Heart Disease
}

Syed KM Gardezi ${ }^{1,4}$, Sean Coffey ${ }^{2}$, Bernard D Prendergast ${ }^{3}$, Saul G Myerson ${ }^{1,4}$

${ }^{1}$ Oxford University Hospitals NHS Foundation Trust, Oxford, United Kingdom

${ }^{2}$ Department of Medicine, Dunedin School of Medicine, University of Otago, New Zealand

${ }^{3}$ St Thomas' Hospital, London, United Kingdom

${ }^{4}$ University of Oxford Radcliffe Department of Medicine, Division of Cardiovascular Medicine, Oxford, United Kingdom

\section{Corresponding author:}

Prof. Saul G Myerson

Department of Cardiology

John Radcliffe Hospital

Oxford OX3 9DU

saul.myerson@cardiov.ox.ac.uk

01865234597

KEYWORDS: Biomarkers, valvular heart disease, left ventricle, aortic stenosis, mitral regurgitation WORD COUNT: 3738 excluding abstract, references

\section{LEARNING OBJECTIVES}

1. Understand the pathophysiological pathways involved in deteriorating valve disease, which may provide useful targets for biomarker development

2. Understand the currently available serum biomarkers in valve disease, and their utility in clinical practice.

3. The potential for future biomarkers, based on the known metabolic and biological pathways 


\section{INTRODUCTION}

Valvular heart disease (VHD) is an important cause of morbidity and mortality whose prevalence is set to increase dramatically as a consequence of the increase in life expectancy in middle- and highincome nations. Community echocardiographic screening has identified a major burden of undiagnosed VHD in older people, with some degree of valve degeneration in almost $50 \%$ and undiagnosed clinically significant (moderate-severe) disease affecting 1 in 10 [1]. Population statistics project an increase in the elderly population, such that there will be an estimated 4 million people aged between 75 and 84 in the UK by 2018, while the population $>85$ years is set to double by 2028.[2] Meanwhile, rheumatic heart disease accounts for a much higher proportion of valve pathology in developing nations and contributes to the growing worldwide prevalence.[3,4]

Despite wider appreciation of the emerging importance of VHD, the mechanisms underlying its development (particularly in degenerative pathology) are poorly understood, and the role of serum biomarkers in guiding clinical management in individual patients is relatively unexplored. European guidelines [5] for the diagnosis and management of VHD make reference to the use of serum B-type natriuretic peptide (BNP) as a marker of prognosis in aortic stenosis (AS) and mitral regurgitation (MR), but thresholds are poorly defined and there are no definitive recommendation on its use.

As the prevalence of VHD increases, it will be increasingly important to develop our understanding of the factors that determine the rate of progression (allowing the planning of careful and efficient follow up and timely intervention). A large proportion of patients with VHD remain asymptomatic and most have mild disease. It is therefore fundamentally important to identify these patients at an earlier stage prior to the development of complications and risk stratify them according to the likelihood of developing significant disease that requires intervention. In this review, we outline current knowledge concerning existing and emerging serum biomarkers that have an established or potential role in the assessment and management of patients with VHD. Imaging (echocardiography, $\mathrm{CT}$ and cardiac magnetic resonance [CMR]) and physiological (e.g. cardiopulmonary exercise testing) are also vital in this clinical endeavour but are beyond the scope of the article. 
The potential roles of circulating biomarkers are:

1. Identification of baseline disease activity and prediction of progression - allows the identification of higher risk patients early, and potentially a subset of patients who might benefit from early surgery (even in the absence of symptoms).

2. Identification of those patients who are less likely to benefit from valve repair/replacement - some have severely impaired left ventricular function that is unlikely to recover after surgery.

3. Understanding the aetiology of VHD - to facilitate the development of future treatment strategies (e.g. tailored medical therapy).

Most existing biomarkers are of myocardial origin (reflecting their established clinical and research pedigree in heart failure) and are likely to provide prognostic utility since morbidity and mortality are largely related to myocardial dysfunction. Conversely, biomarkers of valve leaflet pathology are lacking as a consequence of the relatively small mass of tissue, the rudimentary blood supply, and current poor understanding of the biological stimuli for the development of degenerative VHD.

Myocardial biomarkers may provide significant advantages over existing imaging techniques. For example, whilst CMR is able to identify discrete and diffuse myocardial fibrosis, current techniques are relatively insensitive and only able to identify diffuse fibrosis once it is significantly advanced. Moreover, identification of myocardial wall stress prior to the development of fibrosis would arguably be of greater clinical value.

Biomarkers capable of predicting VHD progression would also be of major clinical value, even if only to determine the frequency of follow-up. For example, the faster rate of progression of calcific aortic stenosis (AS) in subjects with renal disease might be monitored via measurement of factors reflecting the systemic process rather than disease activity affecting the valve itself.

We have focussed on the circulating biomarkers for which reasonable data exists. Several other biomarkers have been examined in an effort to understand the underlying biology of VHD (e.g. serum fetuin-A) [6] but these are generally small cohorts with limited or no clinical follow-up and are not discussed further here.

\section{PATHOPHYSIOLOGY}

Since existing serum biomarkers associated with VHD are mostly related to secondary effects on the ventricular myocardium, a brief overview of the myocardial effects of VHD is helpful. Figure 1 (adapted from reference [7]) also highlights the myocardial physiological processes that may be suitable targets for biomarkers.

\section{A) PRESSURE OVERLOAD IN AORTIC STENOSIS}

Left ventricular systolic pressure is elevated in AS to overcome outflow obstruction with resulting myocyte hypertrophy (to reduce wall stress) and eventual interstitial fibrosis. [8] However, the overall pattern of this adaptive response is highly heterogeneous and may include concentric remodelling and concentric or eccentric hypertrophy.[8] Patterns of left ventricular adaptation and 
degree of hypertrophy correlate poorly with the severity of valve narrowing and asymmetric patterns of wall thickening are common.[9] Left ventricular hypertrophy also leads to reduced density of coronary arteriolar vessels and increased transmural pressures, resulting in increased coronary vascular resistance and reduced coronary flow reserve.[10, 11] The reduction of coronary flow reserve limits the ability of the coronary circulation to increase flow to match myocardial oxygen demand, especially during exercise, and is a key factor in the development of myocardial ischaemia and the occurrence of symptoms.[12] Repetitive myocardial ischaemia related to the exhaustion of coronary flow reserve can lead to apoptosis of myocytes and to the development of 'replacement' myocardial fibrosis. This type of fibrosis occurs predominantly in the subendocardial and mid-wall layers of the left ventricle wall and is generally not reversible following relief of left ventricular pressure overload by AVR.[12] Diastolic dysfunction occurs early in the disease course and worsens with progression of stenosis severity and myocardial fibrosis. In more advanced stages, raised left ventricular filling pressures lead to secondary pulmonary hypertension. $[13,14]$ LV systolic function, measured by ejection fraction (LVEF) and cardiac output, are generally well preserved even in the presence of severe AS, likely due to the increase in LV wall thickness which normalises wall stress whereas the reduced LVEF or cardiac output occurs only in late-stage disease and is usually preceded by clinical symptoms [8]. Subtle LV systolic dysfunction may occur prior to a reduction in ejection fraction however - the occurrence of myocardial fibrosis initially in the subendocardium, in which the myocardial fibres are oriented longitudinally, may result in a reduction in longitudinal function which is compensated for by relatively well preserved radial and circumferential function. $[15,16,17]$ Measures of long axis function (e.g. systolic mitral annular velocities on tissue Doppler echocardiography) may be an indicator of subclinical left ventricular systolic dysfunction despite preserved LVEF and the absence of symptoms, and can be related to adverse outcomes. [18]

The factors that determine the 'tipping' point at which myocardial compensation becomes dysfunction are not entirely clear, and are likely to be variable between individuals. Left ventricular mass does not seem to correlate with the severity of valve disease [9], but has shown some association with future events $[19,20]$, albeit with significant overlap between LV mass groups. The processes of myocyte stress and ultimately degeneration to necrosis/cell death and replacement fibrosis, seem to be better indicators of decompensation, and are associated with reduced LV function on histological assessment of cell degeneration and biochemical/DNA markers [21]. Patchy fibrosis is also a predictor of mortality.[22] These would therefore seem to be suitable targets for biomarker development.

\section{B) VOLUME OVERLOAD IN AORTIC AND MITRAL REGURGITATION}

The left ventricle is volume overloaded in aortic regurgitation (AR) as a result of combined elevation of preload and afterload. The excess preload reflects the volume overload that is in turn directly related to the severity of AR. Afterload is increased since the increased stroke volume is ejected into the high-impedance aorta and systolic hypertension is a frequent consequence. In addition, elevated end-diastolic volume increases LV wall stress. Combined elevation of preload and afterload excess ultimately leads to progressive left ventricular dilatation with resulting systolic dysfunction.[23, 24, $25,26,27]$

The left ventricle is also volume overloaded in mitral regurgitation (MR) though afterload is normal. The adaptive changes of the ventricle include dilatation with normal wall thickness and proportional ('eccentric') hypertrophy [23, 24, 25, 26, 27] while the left atrium also enlarges to accommodate the 
regurgitant volume.[28] Left ventricular ejection fraction may be supra-normal in chronic MR as a result of increased preload and the afterload-reducing effects of ventricular ejection into the lowimpedance left atrium. A low normal ejection fraction can therefore be misleading as a measure of contractile function.[29] Severe MR also leads to pulmonary hypertension (associated with worse outcomes) [30] right ventricular pressure overload and ultimate right ventricular failure.[30] The precise mechanism underlying myocardial decompensation in aortic and mitral regurgitation remains unclear, however it is likely to be related to chronic volume overload leading to eccentric hypertrophy and progressive LV dilatation leading to LV impairment [31].

\section{C) RIGHT VENTRICULAR EFFECTS OF MITRAL STENOSIS AND TRICUSPID REGURGITATION}

Left atrial pressure is increased and compliance is reduced in mitral stenosis (MS) leading to transmission of the trans-mitral pressure gradient to the pulmonary circulation and eventual pulmonary hypertension.[32] Severe MS is associated with pulmonary arteriolar vasoconstriction, intimal hyperplasia and medial hypertrophy and median survival less than 3 years once severe pulmonary hypertension is established.[23, 33]

Tricuspid regurgitation (TR) is usually a secondary phenomenon as a consequence of left sided heart disease or intrinsic pulmonary pathology, rather than resulting from a primary tricuspid valve lesion. Secondary TR is mainly caused by dilation of the tricuspid annulus and/or tethering of the valve leaflets secondary to right ventricular dysfunction. The right ventricle is very tolerant of increasing volume overload well and TR can remain clinically silent for a prolonged period before eventual progressive right ventricular dilatation and dysfunction.[34]

\section{BIOMARKERS ASSOCIATED WITH MYOCARDIAL STRESS}

\section{NATRIURETIC PEPTIDES}

Natriuretic peptides are the most widely used markers of myocardial strain and are primarily synthesized in the heart and regulated by myocardial stress in response to volume or pressure overload [35]. They include B-type natriuretic peptide (BNP) and the N-terminal fragment of its prohormone (NT-proBNP), atrial natriuretic peptide (ANP), adrenomedullin and the mid-regional fragment of the prohormone (MR-proANP). These prohormones are released under conditions of hemodynamic stress and processed into biologically active natriuretic peptides which induce vasodilation, natriuresis and diuresis.[36]

Various studies have demonstrated that BNP is a marker of AS severity and a predictor of poor prognosis in both symptomatic and asymptomatic patients [37, 38, 39, 40, 41, 42] (Table-1). The underlying mechanism triggering BNP activation and release seem to be chronic pressure overload leading to left ventricular hypertrophy, increased wall stress, fibrosis, diastolic dysfunction and raised filling pressures [43]. However, increasing wall thickness/hypertrophy normalises wall stress and may limit the rise in BNP until late in the disease process. In addition, BNP is related to age and there is considerable overlap between different groups of patients with AS, which further limit its utility in clinical practice.

Aortic regurgitation usually progresses slowly with increasing volume overload and left ventricular adaptation by means of dilatation and hypertrophy. Elevation of BNP levels has been associated with severe AR and left ventricular dysfunction on exercise echocardiography, as reflected by a higher 
end-systolic volume index and lower longitudinal strain rate [44], but again this is a late stage of the disease. Various well designed studies have shown that NT-proBNP levels correlate closely with the severity of AR and the patient's functional status [44, 45, 46, 47] (Table-1), but with significant overlap between groups limiting individual utility.

In mitral regurgitation, plasma natriuretic peptide levels increase with increasing severity in patients with both asymptomatic and symptomatic mitral regurgitation, independent of left ventricular systolic function $[48,49]$ and are also associated with higher mortality and the combined end point of death or heart failure in both chronic organic (primary) as well as degenerative MR [50], [51], [52] BNP levels relate to the both the severity as well as being a marker of poor prognosis in patients with MS. $[53,54]$

Data on BNP assays in TR remain limited. In a small study of patients undergoing surgery for severe isolated TR, BNP levels correlated directly with right ventricular volume (and inversely with left ventricular ejection fraction) and were helpful in predicting one year mortality.[55]

In summary, BNP is a potential marker of disease severity and overall prognosis within groups, in patients with AS, AR and MR. However, thresholds of abnormality (which might trigger clinical concern or the need for intervention) are poorly defined and there is significant overlap between groups in the studies to date. Moreover, BNP levels may be elevated in various other conditions, including renal failure, chronic obstructive airways disease, obesity, atrial fibrillation, liver cirrhosis, myocardial infarction, and vary according to age, exercise and fluid status.[56] These factors likely limit its potential for the assessment of individual patients and serial measurement following the definition of baseline levels remains inappropriate until further data emerge.

\section{GROWTH DIFFERENTIATION FACTOR (GDF)-15}

The cytokine GDF-15 (also known as MIC-1) is a member of the transforming growth factor (TGF)- $b$ superfamily [57] and produced at low levels via autocrine or paracrine pathways under baseline conditions by most tissues. $[57,58]$ It provides endogenous protection against ischemia and reperfusion-induced cardiomyocyte apoptosis and is strongly induced via PI3K-Akt-dependent signalling pathways following ischaemic myocardial injury.[59] GDF-15 has been associated with heart failure with preserved ejection fraction and with the degree of myocardial fibrosis in patients with end-stage non-ischaemic dilated cardiomyopathy.[60, 61, 62] Various studies have confirmed its role as a useful predictor of both cardiovascular and all-cause mortality. $[63,64,65,66]$

\section{BIOMARKERS ASSOCIATED WITH MYOCARDIAL HYPERTROPHY AND FIBROSIS}

ST2

ST2 is an inflammatory cytokine member of the interleukin (IL-1) receptor family that predicts mortality and heart failure in patients with acute myocardial infarction and may play a vital role in cardiac pathophysiology. ST2 is thought to be involved in modifying immunologic processes, specifically mediated by T-helper lymphocytes. Interleukin-33, a hormone which may protect against left ventricular hypertrophy and myocardial fibrosis has recently been identified as the ligand for ST2.[36]. In the Framingham Study, ST2 was associated with death, heart failure and major cardiovascular events, but not with echocardiographic indices of myocardial dysfunction. Furthermore, patients with elevated levels of both ST2 and BNP were found to be at considerably 
higher risk of death than those with elevation of one or neither marker.[57] In the emergency setting, the PRIDE study has also found ST2 levels to be higher in patients presenting with acute heart failure [67].

As indicated above, ST2 is one of several markers (alongside GDF-15 \& NTproBNP) which are elevated in patients with severe calcific aortic stenosis and which predict higher mortality after valve replacement.[68] Furthermore, in a recent study of 86 patients with moderate to severe AS (aortic valve area $<1.5 \mathrm{~cm}^{2}$ ) and preserved left ventricular ejection fraction (>50\%), ST2 was related to the severity of valve stenosis the extent of diastolic dysfunction and the ability to distinguish between symptomatic and asymptomatic patients.[69] (Table-1)

ST2 is a promising serum biomarker which correlates well with the severity of AS and poor clinical outcomes. Large prospective studies are now required to validate its utility in patients with significant mitral valve disease.

\section{GALECTIN 3}

Galectin-3 (Gal-3) is a 26-kDa, 3-galactoside-binding lectin [57] found in a wide variety of cells and tissue surfaces which is thought to represent a link between inflammation and fibrosis. Galectin-3 is secreted by activated macrophages, especially at sites of fibrosis and fibroblast deposition, [58] and may therefore play a role in cardiac pathophysiology and act as a surrogate indicator of cardiac remodelling and fibrosis. Expression appears to occur before evidence of heart failure is established and it may therefore be a particularly useful marker in strategies to predict and prevent advanced disease [36].

Serum galectin-3 levels are increased in myocardial biopsies from AS patients with reduced ejection fraction as well as being associated with adverse outcomes after TAVI [70, 71]. Raised galectin-3 levels are also detected in the serum of patients with degenerative mitral valve disease.[72] In contrast, galectin-3 was not associated with AS severity or functional status in a large, single centre, population cohort study (COFRASA-GENERAC) of 583 patients with AS over a wide range of severity.[73] However, one of the limitations of this study was that a large number of participants had only mild or moderate AS.

Interestingly, in vitro and preclinical in vivo studies suggest that Gal-3 may have a role in the disease process at the valve level in AS,[74] but further studies are required to examine this further.

\section{MICRORNA}

MicroRNAs (miRNA) are short (approximately 21 nucleotide) non-protein coding RNAs which are responsible for altering the expression of approximately $30 \%$ of the human genome. $[75,76]$ miRNAs are highly stable in the circulation, either packaged in extracellular vesicles or micro-particles, or bound to molecules such as Argonaute-2. molecules are released into plasma following the liberation of cell contents secondary to necrosis where they remain highly stable [76] and therefore provide an excellent serum marker. Some have tissue specific expression and play a significant role in cellular growth, proliferation and apoptosis. Significant reduction in myocardial hypertrophy was observed following alteration in the expression of miR-21 by gene knockdown, or antisensemediated depletion [77] suggesting that miR-21 is involved in the process of myocardial hypertrophy and may be a possible therapeutic target[76] (Figure-1) . miR-122 levels were found to be down regulated whereas miR-29c, miR-125b \& miR-21 levels were found to be upregulated in studies 
involving patients with AS [78, 79, 80, 81]. In a prospective study of 74 patients with severe AS undergoing AVR surgery preoperative plasma levels of miR-133a were found to predict the regression potential of LV hypertrophy after AVR[82]. However, these small studies rarely take major confounding factors into account, and another study found limited reproducibility once stratified by history of coronary artery disease [83].

\section{BIOMARKERS ASSOCIATED WITH MYOCARDIAL CELL DAMAGE/NECROSIS}

\section{TROPONIN}

Cardiac troponins would seem a good choice of biomarker to explore, given their release into the circulation only after myocardial cell death - one of the potential mechanisms of decompensation identified earlier. However, minor elevation of cardiac troponin I is relatively common in patients with AS (even those with normal coronary arteries) and is conceivably related to higher left ventricular wall thickness and pulmonary artery systolic pressure.[84] Elevated levels of high sensitivity cardiac troponin I \& $\mathrm{T}$ ( $\mathrm{hs} \mathrm{Tnl} / \mathrm{TnT}$ ) have been associated with advanced left ventricular hypertrophy, ECG strain, replacement myocardial fibrosis, and the need for aortic valve replacement as well as predicting survival after transcatheter aortic valve implantation, poor prognosis in patients with AS, and the occurrence of post-operative fatal arrhythmia and cardiac death $[85,86,87$, 88],[89], although the latter three groups involve patients who have already developed symptoms and therefore would not support the use of troponin as an earlier marker of decompensation. Troponins therefore appear to be promising biomarkers to evaluate prognosis in VHD although the overlap between groups is significant, and combining these markers with other factors may help [90]. Larger prospective studies are needed for further validation before incorporation into clinical practice.

\section{METABOLOMICS}

Metabolomics is the systematic study of small molecules in biological fluids [91, 92, 93]. In general, these are assessed in a broad-based fashion, incorporating numerous potential metabolic molecules, and employing advanced statistical methods to identify the signature metabolic profiles in particular cohorts [94] . In this manner, metabolomics has the potential to elucidate poorly understood pathophysiological pathways, identify potential therapeutic targets, and facilitate risk-stratification in individual patients [94]. The metabolic requirements of the myocardium make it feasible that circulating metabolomic profiling could allow quantification of degree of pathological volume or pressure overload in valvular disease, but current knowledge is limited by small sample numbers [95].

\section{GENOMIC BIOMARKERS}

Data from a genome-wide association study including patients from the Cohorts for Heart and Aging Research in Genomic Epidemiology (CHARGE) consortium identified a single-nucleotide polymorphism in the lipoprotein(a) locus which significantly correlated with aortic valve calcification [96]. These findings were subsequently confirmed for clinical aortic stenosis [97]. Another multicentre study involving 220 patients with mild-to-moderate AS showed that elevated oxidized phospholipids on apolipoprotein B-100 (OxPL-apoB) and Lp(a) levels were independently associated with an increased risk of echocardiographically determined AS progression rate, translating to a higher need for AVR accentuated among younger patients.[98] Previous randomised controlled 
trials and a recently published meta-analysis (including 5 randomised control trials and 9 observational studies) have shown no benefit of statins in aortic stenosis [99], but these genomic and serum studies suggest that trials of therapies likely to increase Lp(a) levels might be more successful.

\section{COMBINING MULTIPLE BIOMARKERS}

It is likely, given the multiple processes involved in myocardial degeneration, that a combination of biomarkers are likely to be more useful than any single target. There are few existing studies of multiple biomarkers in VHD, and this remains an area for research. One study in AS examined three biomarkers (GDF-15, ST2 and NTproBNP) in a prospective cohort of 345 intermediate or high risk surgical patients with severe AS undergoing surgical or percutaneous valve replacement. Individual elevated biomarker levels were associated with higher mortality, elevation of all three biomarkers demonstrated a stronger predictive ability, with a 10-fold higher 1- and 2-year mortality than those with normal levels. [68] A multimarker approach representing diverse biological pathways thus seems likely to be more beneficial.

\section{CONCLUSION}

Established biomarkers such as BNP and high sensitivity troponin can play a partial role in predicting the progression of VHD in patient groups. However, overlap between groups with differing severity and prognosis is significant and further research will be necessary to determine if meaningful clinical thresholds exist for individual patients. Emerging biomarkers, in particular mi-RNA, GDF-15, ST2, Galectin-3 and DNA profiling, hold promise for risk stratification and tailored disease management. Ultimately, it is likely that a combination of biomarkers will be more useful than a single factor, and the development of risk scores combining the serum biomarkers and other parameters, such as cardiac imaging may prove most beneficial in improving risk stratification in VHD. 


\section{KEY POINTS}

1. The likely processes involved in the transition from compensated to decompensated valve disease include cardiomyocyte stress, apoptosis/cell death and replacement fibrosis, which form suitable targets for biomarker development.

2. Natriuretic peptides are the most widely studied biomarkers in valve disease, but they are not specific to valve disease and there is considerable overlap in serum levels between different clinical groups.

3. Combinations of biomarkers are likely to prove more beneficial than any single marker, and have been successfully utilised in other disease areas, and to a limited extent in valve disease

4. Aortic stenosis is the most widely researched individual valve lesion, and useful biomarkers for predicting prognosis include a combination of ST2, GDF-15 \& NTproBNP, in addition to highsensitivity troponins.

5. Future biomarkers will likely include micro-RNAs and metabolomic markers, as more specific markers of valve decompensation

\section{COMPETING INTERESTS}

The authors have no competing interests

\section{FUNDING}

National Institute for Health Research (NIHR) Oxford Biomedical Research Centre

\section{LICENSE}

The Corresponding Author has the right to grant on behalf of all authors and does grant on behalf of all authors, an exclusive licence (or non exclusive for government employees) on a worldwide basis to the BMJ Publishing Group Ltd and its Licensees to permit this article (if accepted) to be published in HEART editions and any other BMJPGL products to exploit all subsidiary rights. 


\section{REFERENCES (key papers in bold)}

1 d'Arcy JL, Coffey S, Loudon MA, et al. Large-scale community echocardiographic screening reveals a major burden of undiagnosed valvular heart disease in older people: the OxVALVE Population Cohort Study. Eur Heart J 2016;37:3515-22.

\section{- Contemporary population based estimate of VHD prevalence in large sample (>4,000 subjects). Major burden of previously undiagnosed VHD}

2 d'Arcy JL, Prendergast BD, Chambers JB, et al. Valvular heart disease: the next cardiac epidemic. Heart 2011;97:91-3.

3 Veinot JP. Pathology of inflammatory native valvular heart disease. Cardiovasc Pathol 2006;15:243-51.

4 Coffey S, Cairns BJ, lung B. The modern epidemiology of heart valve disease. Heart 2016;102:7585.

5 Joint Task Force on the Management of Valvular Heart Disease of the European Society of C, European Association for Cardio-Thoracic S, Vahanian A, et al. Guidelines on the management of valvular heart disease (version 2012). Eur Heart J 2012;33:2451-96.

$6 \mathrm{Ix} \mathrm{JH}$, Chertow GM, Shlipak MG, et al. Association of Fetuin-A With Mitral Annular Calcification and Aortic Stenosis Among Persons With Coronary Heart Disease. Circulation 2007;115:2533.

7 Thum T. MicroRNA therapeutics in cardiovascular medicine. EMBO Mol Med 2012;4:3-14.

8 Lindman BR, Clavel MA, Mathieu P, et al. Calcific aortic stenosis. Nat Rev Dis Primers 2016;2:16006.

9 Dweck MR, Joshi S, Murigu T, et al. Left ventricular remodeling and hypertrophy in patients with aortic stenosis: insights from cardiovascular magnetic resonance. J Cardiovasc Magn Reson 2012;14:50.

10 Rajappan K, Rimoldi OE, Camici PG, et al. Functional changes in coronary microcirculation after valve replacement in patients with aortic stenosis. Circulation 2003;107:3170-5.

11 Rajappan K, Rimoldi OE, Dutka DP, et al. Mechanisms of Coronary Microcirculatory Dysfunction in Patients With Aortic Stenosis and Angiographically Normal Coronary Arteries. Circulation 2002;105:470-6.

12 Julius BK, Spillmann M, Vassalli G, et al. Angina pectoris in patients with aortic stenosis and normal coronary arteries. Mechanisms and pathophysiological concepts. Circulation 1997;95:892-8.

13 Mutlak D, Aronson D, Carasso S, et al. Frequency, Determinants and Outcome of Pulmonary Hypertension in Patients With Aortic Valve Stenosis. The American Journal of the Medical Sciences;343:397-401.

14 Lancellotti P, Magne J, Donal E, et al. Determinants and prognostic significance of exercise pulmonary hypertension in asymptomatic severe aortic stenosis. Circulation 2012;126:851-9.

15 Dumesnil JG, Shoucri RM, Laurenceau JL, et al. A mathematical model of the dynamic geometry of the intact left ventricle and its application to clinical data. Circulation 1979;59:1024-34.

16 Lancellotti P, Donal E, Magne J, et al. Risk stratification in asymptomatic moderate to severe aortic stenosis: the importance of the valvular, arterial and ventricular interplay. Heart 2010;96:1364-71.

17 Kusunose K, Goodman A, Parikh R, et al. Incremental prognostic value of left ventricular global longitudinal strain in patients with aortic stenosis and preserved ejection fraction. Circ Cardiovasc Imaging 2014;7:938-45. 
18 Carstensen HG, Larsen LH, Hassager C, et al. Tissue Velocities and Myocardial Deformation in Asymptomatic and Symptomatic Aortic Stenosis. J Am Soc Echocardiogr 2015;28:969-80.

19 Cioffi G, Faggiano P, Vizzardi E, et al. Prognostic effect of inappropriately high left ventricular mass in asymptomatic severe aortic stenosis. Heart 2011;97:301.

20 Gerdts E, Rossebo AB, Pedersen TR, et al. Relation of Left Ventricular Mass to Prognosis in Initially Asymptomatic Mild to Moderate Aortic Valve Stenosis. Circ Cardiovasc Imaging 2015;8:e003644; discussion e.

21 Hein S, Arnon E, Kostin S, et al. Progression from compensated hypertrophy to failure in the pressure-overloaded human heart: structural deterioration and compensatory mechanisms. Circulation 2003;107:984-91.

22 Dweck MR, Joshi S, Murigu T, et al. Midwall fibrosis is an independent predictor of mortality in patients with aortic stenosis. J Am Coll Cardiol 2011;58:1271-9.

23 Maganti K, Rigolin VH, Sarano ME, et al. Valvular heart disease: diagnosis and management. Mayo Clin Proc 2010;85:483-500.

24 Gaasch WH, Zile MR. Left Ventricular Structural Remodeling in Health and Disease. J Am Coll Cardiol 2011;58:1733.

25 Carabello BA. Progress in mitral and aortic regurgitation. Prog Cardiovasc Dis 2001;43:457-75.

26 Borer JS, Bonow RO. Contemporary Approach to Aortic and Mitral Regurgitation. Circulation 2003;108:2432.

27 Rigolin VH, Bonow RO. Hemodynamic characteristics and progression to heart failure in regurgitant lesions. Heart Fail Clin 2006;2:453-60.

28 Borer JS, Bonow RO. Contemporary Approach to Aortic and Mitral Regurgitation. Circulation 2003;108:2432-8.

29 Enriquez-Sarano M, Avierinos J-F, Messika-Zeitoun D, et al. Quantitative Determinants of the Outcome of Asymptomatic Mitral Regurgitation. N Engl J Med 2005;352:875-83.

30 Stone GW, Vahanian AS, Adams DH, et al. Clinical Trial Design Principles and Endpoint Definitions for Transcatheter Mitral Valve Repair and Replacement: Part 1: Clinical Trial Design Principles: A Consensus Document From the Mitral Valve Academic Research Consortium. J Am Coll Cardiol 2015;66:278-307.

31 Ahmed MI, Gladden JD, Litovsky SH, et al. Increased Oxidative Stress and Cardiomyocyte Myofibrillar Degeneration in Patients with Chronic Isolated Mitral Regurgitation and Ejection Fraction > 60\%. J Am Coll Cardiol 2010;55:671-9.

$32 \mathrm{Kim} \mathrm{HK}, \mathrm{Kim} \mathrm{YJ}$, Shin Jl, et al. Echocardiographic and hemodynamic findings in patients with mitral stenosis undergoing percutaneous mitral commissurotomy comparing those with chronic atrial fibrillation versus those with normal sinus rhythm. Am J Cardiol 2007;100:1153-6.

33 Bonow RO, Carabello BA, Chatterjee K, et al. 2008 Focused update incorporated into the ACC/AHA 2006 guidelines for the management of patients with valvular heart disease: a report of the American College of Cardiology/American Heart Association Task Force on Practice Guidelines (Writing Committee to Revise the 1998 Guidelines for the Management of Patients With Valvular Heart Disease): endorsed by the Society of Cardiovascular Anesthesiologists, Society for Cardiovascular Angiography and Interventions, and Society of Thoracic Surgeons. Circulation 2008;118:e523-661.

34 Lancellotti P, Fattouch K, Dulgheru R. Targeting the tricuspid valve: A new therapeutic challenge. Arch Cardiovasc Dis 2016;109:1-3.

35 Mair J, Jaffe A, Apple F, et al. Cardiac biomarkers. Dis Markers 2015;2015:370569. 
36 Iqbal N, Wentworth B, Choudhary R, et al. Cardiac biomarkers: new tools for heart failure management. Cardiovasc Diagn Ther 2012;2:147-64.

37 Gerber IL, Stewart RA, Legget ME, et al. Increased plasma natriuretic peptide levels reflect symptom onset in aortic stenosis. Circulation 2003;107:1884-90.

38 Henri C, Dulgheru R, Magne J, et al. Impact of Serial B-Type Natriuretic Peptide Changes for Predicting Outcome in Asymptomatic Patients With Aortic Stenosis. Can J Cardiol 2016;32:1839.

39 Clavel MA, Malouf J, Michelena $\mathrm{HI}$, et al. B-type natriuretic peptide clinical activation in aortic stenosis: impact on long-term survival. J Am Coll Cardiol 2014;63:2016-25.

\section{- Key paper on BNP in aortic stenosis, related to prognosis}

40 Monin J-L, Lancellotti P, Monchi M, et al. Risk Score for Predicting Outcome in Patients With Asymptomatic Aortic Stenosis. Circulation 2009;120:69.

41 Nessmith MG, Fukuta H, Brucks S, et al. Usefulness of an elevated B-type natriuretic peptide in predicting survival in patients with aortic stenosis treated without surgery. Am J Cardiol 2005;96:1445-8.

42 Bergler-Klein J, Klaar U, Heger M, et al. Natriuretic Peptides Predict Symptom-Free Survival and Postoperative Outcome in Severe Aortic Stenosis. Circulation 2004;109:2302.

43 Genereux P, Stone GW, O'Gara PT, et al. Natural History, Diagnostic Approaches, and Therapeutic Strategies for Patients With Asymptomatic Severe Aortic Stenosis. J Am Coll Cardiol 2016;67:2263-88.

44 Gabriel RS, Kerr AJ, Sharma V, et al. B-type natriuretic peptide and left ventricular dysfunction on exercise echocardiography in patients with chronic aortic regurgitation. Heart 2008;94:897902.

45 Pizarro R, Bazzino OO, Oberti PF, et al. Prospective validation of the prognostic usefulness of Btype natriuretic peptide in asymptomatic patients with chronic severe aortic regurgitation. $J \mathrm{Am}$ Coll Cardiol 2011;58:1705-14.

46 Weber M, Hausen M, Arnold R, et al. Diagnostic and prognostic value of $\mathrm{N}$-terminal pro B-type natriuretic peptide (NT-proBNP) in patients with chronic aortic regurgitation. Int J Cardiol 2008;127:321-7.

47 Gerber IL, Stewart RA, French JK, et al. Associations between plasma natriuretic peptide levels, symptoms, and left ventricular function in patients with chronic aortic regurgitation. $A m \mathrm{~J}$ Cardiol 2003;92:755-8.

48 Sutton TM, Stewart RAH, Gerber IL, et al. Plasma natriuretic peptide levels increase with symptoms and severity of mitral regurgitation. J Am Coll Cardiol 2003;41:2280.

49 Pizarro R, Bazzino O0, Oberti PF, et al. Prospective validation of the prognostic usefulness of brain natriuretic peptide in asymptomatic patients with chronic severe mitral regurgitation. $J$ Am Coll Cardiol 2009;54:1099-106.

- Key paper on BNP in mitral regurgitation

50 Detaint D, Messika-Zeitoun D, Avierinos JF, et al. B-type natriuretic peptide in organic mitral regurgitation: determinants and impact on outcome. Circulation 2005;111:2391-7.

51 Wohrle J, Karakas M, Trepte U, et al. Midregional-proAtrial Natriuretic Peptide and High Sensitive Troponin T Strongly Predict Adverse Outcome in Patients Undergoing Percutaneous Repair of Mitral Valve Regurgitation. PLoS One 2015;10:e0137464. 
52 Clavel M-A, Tribouilloy C, Vanoverschelde J-L, et al. Association of B-Type Natriuretic Peptide With Survival in Patients With Degenerative Mitral Regurgitation. J Am Coll Cardiol 2016;68:1297-307.

53 Eryol NK, Dogan A, Ozdogru I, et al. The relationship between the level of plasma B-type natriuretic peptide and mitral stenosis. Int J Cardiovasc Imaging 2007;23:569-74.

54 Sharma V, Stewart RA, Zeng I, et al. Comparison of atrial and brain natriuretic peptide for the assessment of mitral stenosis. Heart Lung Circ 2011;20:517-24.

55 Yoon $\mathrm{CH}$, Zo JH, Kim YJ, et al. B-type natriuretic Peptide in isolated severe tricuspid regurgitation: determinants and impact on outcome. J Cardiovasc Ultrasound 2010;18:139-45.

56 Vassiliadis E, Barascuk N, Didangelos A, et al. Novel cardiac-specific biomarkers and the cardiovascular continuum. Biomark Insights 2012;7:45-57.

57 Collinson P. The role of cardiac biomarkers in cardiovascular disease risk assessment. Curr Opin Cardiol 2014;29:366-71.

58 Loncar G, Omersa D, Cvetinovic N, et al. Emerging biomarkers in heart failure and cardiac cachexia. Int J Mol Sci 2014;15:23878-96.

59 Kempf T, Eden M, Strelau J, et al. The transforming growth factor-beta superfamily member growth-differentiation factor-15 protects the heart from ischemia/reperfusion injury. Circ Res 2006;98:351-60.

60 Santhanakrishnan R, Chong JP, Ng TP, et al. Growth differentiation factor 15, ST2, highsensitivity troponin $\mathrm{T}$, and $\mathrm{N}$-terminal pro brain natriuretic peptide in heart failure with preserved vs. reduced ejection fraction. Eur J Heart Fail 2012;14:1338-47.

61 Lok SI, Winkens B, Goldschmeding R, et al. Circulating growth differentiation factor-15 correlates with myocardial fibrosis in patients with non-ischaemic dilated cardiomyopathy and decreases rapidly after left ventricular assist device support. Eur J Heart Fail 2012;14:1249-56.

62 Stahrenberg R, Edelmann F, Mende M, et al. The novel biomarker growth differentiation factor 15 in heart failure with normal ejection fraction. Eur J Heart Fail 2010;12:1309-16.

63 Eggers KM, Kempf T, Wallentin L, et al. Change in growth differentiation factor 15 concentrations over time independently predicts mortality in community-dwelling elderly individuals. Clin Chem 2013;59:1091-8.

64 Wallentin L, Zethelius B, Berglund L, et al. GDF-15 for prognostication of cardiovascular and cancer morbidity and mortality in men. PLoS One 2013;8:e78797.

65 Xanthakis V, Larson MG, Wollert KC, et al. Association of novel biomarkers of cardiovascular stress with left ventricular hypertrophy and dysfunction: implications for screening. J Am Heart Assoc 2013;2:e000399.

66 Heringlake M, Charitos El, Gatz N, et al. Growth differentiation factor 15: a novel risk marker adjunct to the EuroSCORE for risk stratification in cardiac surgery patients. J Am Coll Cardiol 2013;61:672-81.

67 Januzzi JL, Jr., Peacock WF, Maisel AS, et al. Measurement of the interleukin family member ST2 in patients with acute dyspnea: results from the PRIDE (Pro-Brain Natriuretic Peptide Investigation of Dyspnea in the Emergency Department) study. J Am Coll Cardiol 2007;50:60713.

68 Lindman BR, Breyley JG, Schilling JD, et al. Prognostic utility of novel biomarkers of cardiovascular stress in patients with aortic stenosis undergoing valve replacement. Heart 2015;101:1382-8. 
- Paper combining three markers to produce stronger predictive value in patients undergoing AVR

69 Lancellotti P, Dulgheru R, Magne J, et al. Elevated Plasma Soluble ST2 Is Associated with Heart Failure Symptoms and Outcome in Aortic Stenosis. PLoS One 2015;10:e0138940.

70 Sharma UC, Pokharel S, van Brakel TJ, et al. Galectin-3 marks activated macrophages in failureprone hypertrophied hearts and contributes to cardiac dysfunction. Circulation 2004;110:31218.

71 Baldenhofer G, Zhang K, Spethmann S, et al. Galectin-3 predicts short- and long-term outcome in patients undergoing transcatheter aortic valve implantation (TAVI). Int J Cardiol 2014;177:912-7.

72 Sakarin S, Rungsipipat A, Surachetpong SD. Galectin-3 in cardiac muscle and circulation of dogs with degenerative mitral valve disease. J Vet Cardiol 2016;18:34-46.

73 Arangalage D, Nguyen V, Robert T, et al. Determinants and prognostic value of Galectin-3 in patients with aortic valve stenosis. Heart 2016;102:862-8.

74 Sadaba JR, Martinez-Martinez E, Arrieta V, et al. Role for Galectin-3 in Calcific Aortic Valve Stenosis. J Am Heart Assoc 2016;5.

75 Devaux $\mathrm{Y}$, Stammet $\mathrm{P}$, Friberg $\mathrm{H}$, et al. MicroRNAs: new biomarkers and therapeutic targets after cardiac arrest? Crit Care 2015;19:54.

76 Jankovic R, Markovic D, Savic N, et al. Beyond the Limits: Clinical Utility of Novel Cardiac Biomarkers. Biomed Res Int 2015;2015:187384.

77 Ji R, Cheng Y, Yue J, et al. MicroRNA expression signature and antisense-mediated depletion reveal an essential role of MicroRNA in vascular neointimal lesion formation. Circ Res 2007;100:1579-88.

78 Beaumont J, Lopez B, Hermida N, et al. microRNA-122 down-regulation may play a role in severe myocardial fibrosis in human aortic stenosis through TGF-beta1 up-regulation. Clin Sci (Lond) 2014;126:497-506.

79 Derda AA, Thum S, Lorenzen JM, et al. Blood-based microRNA signatures differentiate various forms of cardiac hypertrophy. Int J Cardiol 2015;196:115-22.

80 Ohukainen P, Syvaranta S, Napankangas J, et al. MicroRNA-125b and chemokine CCL4 expression are associated with calcific aortic valve disease. Ann Med 2015;47:423-9.

81 Villar AV, Garcia R, Merino D, et al. Myocardial and circulating levels of microRNA-21 reflect left ventricular fibrosis in aortic stenosis patients. Int J Cardiol 2013;167:2875-81.

- helpful paper linking microRNA and aortic stenosis - potential mechanistic insights

82 Garcia R, Villar AV, Cobo M, et al. Circulating levels of miR-133a predict the regression potential of left ventricular hypertrophy after valve replacement surgery in patients with aortic stenosis. $J$ Am Heart Assoc 2013;2:e000211.

83 Coffey S, Williams MJ, Phillips LV, et al. Circulating microRNA Profiling Needs Further Refinement Before Clinical Use in Patients With Aortic Stenosis. J Am Heart Assoc 2015;4:e002150.

84 Nunes JPL, Mota Garcia JM, Farinha RMB, et al. Cardiac troponin I in aortic valve disease. Int J Cardiol 2003;89:281-5.

85 Rosjo H, Andreassen J, Edvardsen T, et al. Prognostic usefulness of circulating high-sensitivity troponin $T$ in aortic stenosis and relation to echocardiographic indexes of cardiac function and anatomy. Am J Cardiol 2011;108:88-91. 
86 Frank D, Stark S, Lutz M, et al. Preprocedural high-sensitive troponin predicts survival after transcatheter aortic valve implantation (TAVI). Int J Cardiol 2013;169:e38-9.

87 Chin CW, Shah AS, McAllister DA, et al. High-sensitivity troponin I concentrations are a marker of an advanced hypertrophic response and adverse outcomes in patients with aortic stenosis. Eur Heart J 2014;35:2312-21.

\section{- important paper on troponin in aortic stenosis}

88 Shah AS, Chin CW, Vassiliou V, et al. Left ventricular hypertrophy with strain and aortic stenosis. Circulation 2014;130:1607-16.

89 Saito $\mathrm{T}$, Hojo $\mathrm{Y}$, Hirose $\mathrm{M}$, et al. High-sensitivity troponin $\mathrm{T}$ is a prognostic marker for patients with aortic stenosis after valve replacement surgery. J Cardiol 2013;61:342-7.

90 Chin CWL, Messika-Zeitoun D, Shah ASV, et al. A clinical risk score of myocardial fibrosis predicts adverse outcomes in aortic stenosis. Eur Heart J 2016;37:713-23.

91 Nicholson JK. Global systems biology, personalized medicine and molecular epidemiology. Mol Syst Biol 2006;2:52.

92 Nicholson JK, Connelly J, Lindon JC, et al. Metabonomics: a platform for studying drug toxicity and gene function. Nat Rev Drug Discov 2002;1:153-61.

93 Nicholson JK, Lindon JC, Holmes E. 'Metabonomics': understanding the metabolic responses of living systems to pathophysiological stimuli via multivariate statistical analysis of biological NMR spectroscopic data. Xenobiotica 1999;29:1181-9.

94 Dona AC, Coffey S, Figtree G. Translational and emerging clinical applications of metabolomics in cardiovascular disease diagnosis and treatment. Eur J Prev Cardiol 2016;23:1578-89.

95 Mourino-Alvarez L, Baldan-Martin M, Gonzalez-Calero L, et al. Patients with calcific aortic stenosis exhibit systemic molecular evidence of ischemia, enhanced coagulation, oxidative stress and impaired cholesterol transport. Int J Cardiol 2016;225:99-106.

96 Thanassoulis G, Campbell CY, Owens DS, et al. Genetic associations with valvular calcification and aortic stenosis. N EnglJ Med 2013;368:503-12.

97 Cairns BJ, Coffey S, Travis RC, et al. A Replicated, Genome-Wide Significant Association of Aortic Stenosis With a Genetic Variant for Lipoprotein(a): Meta-Analysis of Published and Novel Data. Circulation 2017;135:1181-3.

98 Capoulade R, Chan KL, Yeang C, et al. Oxidized Phospholipids, Lipoprotein(a), and Progression of Calcific Aortic Valve Stenosis. J Am Coll Cardiol 2015;66:1236-46.

99 Zhao Y, Nicoll R, He Yh, et al. The effect of statins therapy in aortic stenosis: Meta-analysis comparison data of RCTs and observationals. Data in Brief 2016;7:357-61. 
Table-1

A summary of the key studies showing the role of serum biomarkers in valvular heart disease

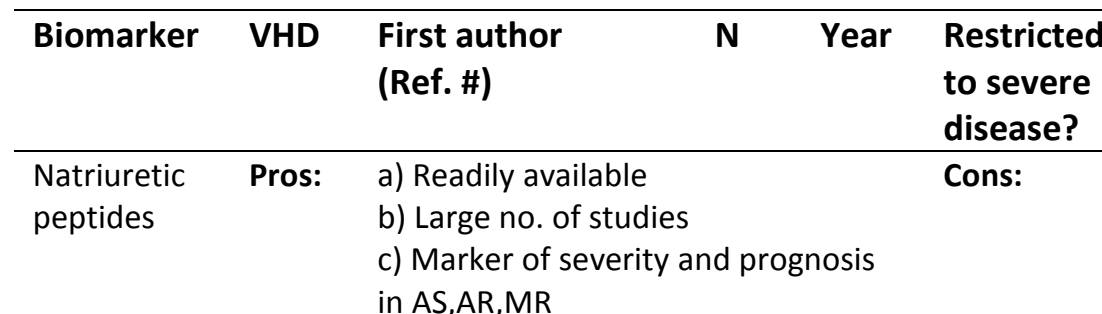

\begin{tabular}{|c|c|c|c|c|}
\hline AS & Gerber et al.[37] & 74 & 2003 & No \\
\hline AS & $\begin{array}{l}\text { Bergler-Klein et } \\
\text { al.[42] }\end{array}$ & 130 & 2004 & Yes \\
\hline AS & Nessmith et al.[41] & 124 & 2005 & No \\
\hline AS & Monin et al.[40] & 107 & 2009 & No \\
\hline AS & Clavel et al.[39] & 1953 & 2014 & Yes \\
\hline AS & Henri et al.[38] & 69 & 2016 & No \\
\hline$A R$ & Gerber et al.[47] & 40 & 2003 & Yes \\
\hline
\end{tabular}

a) Poorly defined thresholds

b) Significant overlap between groups

c) Lacks specificity for VHD - can be elevated in other unrelated medical conditions
NT-proBNP and BNP both associated with symptoms (AUC: 0.84 and 0.83 , respectively)

NT-proBNP and BNP both predicted the presence of symptoms as well as the risk of symptom onset or death among subjects with severe AS

BNP predicted presence of symptoms (AUC: 0.87) Optimal cut off was $190 \mathrm{pg} / \mathrm{ml}$

BNP independently predicted outcomes (cardiac death, hospitalization for CHF, or AVR)

BNP independently predicted long term mortality even among asymptomatic subjects with at least moderate aortic stenosis

Annual change in BNP levels predicted outcomes (symptoms, AVR, or death)

Natriuretic peptide (ANP,BNP \& N-BNP) levels correspond with symptomatic status among patients with moderate to severe AR and preserved LV systolic function

Increased BNP level associated with increased severity of regurgitation \& early LV dysfunction on exercise echo among asymptomatic or mildly symptomatic patients with moderate to severe AR with preserved LV systolic function

NT-proBNP linked to disease severity and functional status. It also predicted prognosis \& clinical outcomes in patients with chronic aortic regurgitation \& preserved LV systolic function

BNP independently predicted prognosis among asymptomatic patients with severe AR and preserved LV systolic function

Natriuretic peptide (BNP, ANP, N-BNP) levels correspond with increasing severity of $M R$ and symptomatic status (AUC 0.9, 0.89, 0.89 respectively) even among patients with preserved LV function 


\begin{tabular}{|c|c|c|c|c|c|}
\hline & & & & & adverse events including death and neart fallure \\
\hline$M R$ & Detaint et al.[50] & 124 & 2005 & No & \\
\hline MR & Pizarro et al.[49] & 269 & 2009 & Yes & $\begin{array}{l}\text { BNP was an independent predictor of adverse events } \\
\text { including heart failure, LV dysfunction and death } \\
\text { among patients with severe asymptomatic MR }\end{array}$ \\
\hline MR & Clavel et al.[52] & 1331 & 2016 & No & $\begin{array}{l}\text { BNP-ratio(ratio to upper limit of normal for age, sex, } \\
\text { and assay) was found to be an independent, and } \\
\text { incremental predictor of long-term mortality in } \\
\text { patients with degenerative MR on medical therapy }\end{array}$ \\
\hline MS & Sharma et al.[54] & 44 & 2011 & Yes & $\begin{array}{l}\text { Raise serum BNP levels were associated with left atrial } \\
\text { dilatation, raised pulmonary artery pressure, reduced } \\
\text { mitral valve area and treadmill exercise capacity as } \\
\text { well as being a marker of adverse events among } \\
\text { patients with moderate to severe MS }\end{array}$ \\
\hline TR & Yoon et al.[55] & 39 & 2010 & Yes & $\begin{array}{l}\text { An elevated BNP level was determined by the LVEF } \\
\text { and RVESVI, a baseline BNP level of }>200 \mathrm{pg} / \mathrm{ml} \\
\text { predicted poor outcomes and increased mortality and } \\
\text { morbidity among patients with isolated, severe TR } \\
\text { undergoing corrective surgery }\end{array}$ \\
\hline
\end{tabular}
BNP was found to be an independent predictor of
adverse events including death and heart failure

BNP was an independent predictor of adverse events including heart failure, LV dysfunction and death BNP-ratio(ratio to upper limit of normal for age, sex, and assay) was found to be an independent, and incremental predictor of long-term mortality in Raise serum BNP levels were associated with left atrial dilatation, raised pulmonary artery pressure, reduced well as being a marker of adverse events among An elevated BNP level was determined by the LVEF and RVESVI, a baseline BNP level of $>200 \mathrm{pg} / \mathrm{ml}$ morbidity among patients with isolated, severe TR undergoing corrective surgery

\begin{tabular}{|c|c|c|}
\hline $\begin{array}{l}\text { GDF-15, } \\
\text { ST2 }\end{array}$ & pros & $\begin{array}{l}\text { a) markers of prognosis in AS } \\
\text { undergoing AVR } \\
\text { b) ST2 reflects symptom status, } \\
\text { severity, diastolic function as well as } \\
\text { being an independent predictor of } \\
\text { cardiovascular events in AS }\end{array}$ \\
\hline
\end{tabular}

cons

a) Limited available data for GDF-15

b) Available data only in AS

AS Lancellotti et al.[69] $86 \quad 2015 \quad$ Yes

\begin{tabular}{|c|}
\hline $\begin{array}{l}\text { Combinatio } \\
\text { n GDF15, } \\
\text { ST2, } \\
\text { NT-proBNP }\end{array}$ \\
\hline
\end{tabular}

ST2 levels were related to symptomatic status, AS severity \& diastolic dysfunction; furthermore ST2 was identified as an independent predictor of cardiovascular events among patients with moderate to severe AS and preserved LV function

Elevated baseline GDF-15, ST2 and NTproBNP levels were associated with a higher post-operative mortality among patients with severe AS undergoing surgical or percutaneous valve replacement. Patients with elevation of all three biomarkers had a 10-fold higher

1- and 2-year mortality than those with normal levels

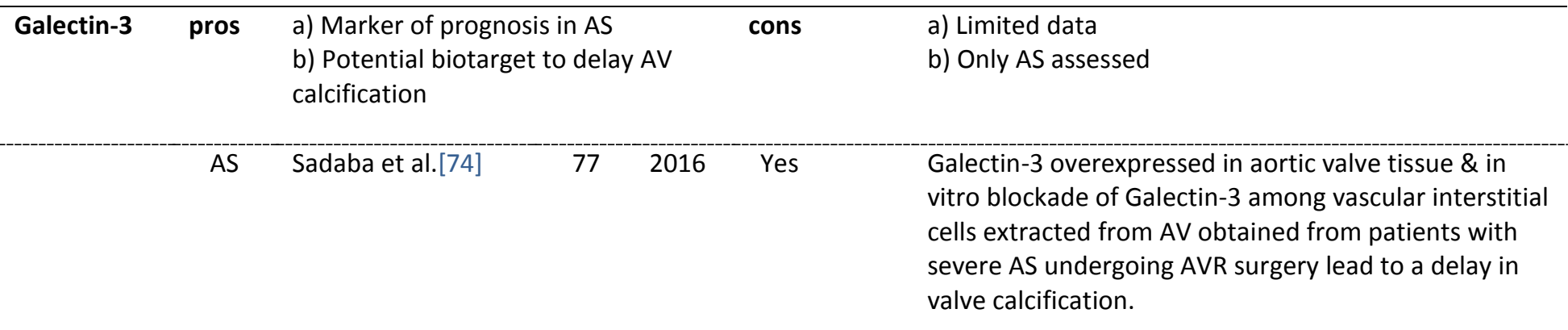




\begin{tabular}{llll}
\hline Troponins $\quad$ pros & a) readily available & cons & a) Not specific for VHD - can be elevated in other \\
& $\begin{array}{ll}\text { b) various large studies } \\
\text { c) marker of prognosis in AS, MR }\end{array}$ & & conditions \\
&
\end{tabular}

AS Rosjo et al.[85] $\quad 57 \quad 2011$ Yes $\quad$ hs-cTnT levels were universally detectable, LV mass
and systolic function were independent determinants of hs-cTnT levels \& high hs-cTnT levels were associated with a poor prognosis among patients with moderate to severe AS

AS Frank et al.[86] $\quad 107 \quad 2013 \quad$ Yes

hsTnT was found to be superior to NTproBNP in predicting survival \& hsTnT alongside pre-procedural AR were identified as the independent predictors of survival among patients with severe AS undergoing TAVI

AS Chin et al.[87] $\quad 253 \quad 2014 \quad$ No

Plasma cTnl concentrations detectable in $98 \%$ of subjects and were increased when compared with age- and sex-matched healthy volunteers. cTnl concentrations were found to be associated with advanced LV hypertrophy and replacement fibrosis as well as AVR or cardiovascular death among patients with AS

AS Shah et al.[88] $\quad 242 \quad 2014 \quad$ No

ECG LVH with strain was identified as a marker of midwall myocardial fibrosis and it was also associated with increased myocardial injury, impaired left ventricular function and was found to be an independent predictor of cardiovascular death or AVR among patients with AS

(Troponins MR Wohrle et al.[51] $\quad 34 \quad 2015 \quad$ Yes
\& MRproANP)
a) Several small studies
cons
b) can help in understanding pathophysiology of VHD c) potential biotarget in myocardial reverse remodelling

MR-proANP \& hsTnT were strong predictors of cardiovascular death and rehospitalization for HF among patients with severe MR undergoing percutaneous repair for MR (MitraClip procedure) 

predict the regression potential of LV hypertrophy post AVR among patients with severe AS undergoing AVR AUC 0.89, p-value $<0.001$

AS Villar et al.[81]

107

2013

Yes

AS Beaumont et al.[78]
Myocardial and circulating levels of miR-21 were higher and correlated directly with the transvalvular gradients among patients with severe AS when compared to controls

miR-122 was found to be down regulated in endomyocardial biopsies taken from 28 patients using necropsies of 10 subjects as controls, possibly leading to myocardial fibrosis through TGF- $\beta 1$ up-regulation among subjects with severe AS

miR-29a levels were found to be increased in patients with hypertrophic obstructive cardiomyopathy while miR-29c were found to be increased in patients with aortic stenosis (awaiting TAVI) in a study involving patients with aortic stenosis and hypertrophic cardiomyopathy

Abbreviations: $\mathrm{miR}=$ microRNA, $\mathrm{TAVI}=$ transcatheter aortic valve intervention, $\mathrm{GDF}=$ Growth differentiation factor, TGF= transforming growth factor, TnI=Troponin-I, TnT=Troponin-T, hs=high sensitivity, MRproANP=mid-regional pro atrial natriuretic peptide, $N T$ proBNP $=\mathrm{N}$-terminal Pro-BNP, N-BNP=N-terminal BNP 